\title{
The impact of elastography with virtual touch quantification of future remnant liver before major hepatectomy
}

\author{
Shingo Shimada ${ }^{1}$, Toshiya Kamiyama ${ }^{1}$, Tatsuhiko Kakisaka ${ }^{1}$, Tatsuya Orimo ${ }^{1}$, Akihisa Nagatsu ${ }^{1}$, \\ Yoh Asahi ${ }^{1}$, Yuzuru Sakamoto ${ }^{1}$, Hirofumi Kamachi ${ }^{1}$, Yusuke Kudo ${ }^{2}$, Mutsumi Nishida ${ }^{2}$, \\ Akinobu Taketomi ${ }^{1}$
}

${ }^{1}$ Department of Gastroenterological Surgery I, Hokkaido University Graduate School of Medicine, Sapporo, Japan; ${ }^{2}$ Division of Laboratory and Transfusion Medicine, Hokkaido University Hospital, Sapporo, Hokkaido, Japan

Correspondence to: Shingo Shimada. Department of Gastroenterological Surgery I, Hokkaido University Graduate School of Medicine, Sapporo, Japan North 15-West 7, Kita-Ku, Sapporo, Hokkaido 060-8638, Japan. Email: shingoshimada1979@true.ocn.ne.jp.

Background: Liver elastography with virtual touch quantification (VTQ) measures the velocity of the shear wave generated by a short-duration acoustic force impulse, with values expressed in units of velocity $(\mathrm{m} / \mathrm{s})$. VTQ can evaluate right or left hepatic lobes separately. VTQ might be appropriate for the evaluation of future remnant liver after hepatectomy.

Methods: We analyzed 95 patients underwent liver elastography with VTQ and both future remnant liver and resected side before hepatectomy of more than two sections, except for central bisectionectomy. We divided the patients into a high VTQ group $(\geq 1.52 \mathrm{~m} / \mathrm{s}, \mathrm{n}=37,39 \%)$ and a low VTQ group $(<1.52 \mathrm{~m} / \mathrm{s}, \mathrm{n}=58$, $61 \%$ ) according to the VTQ of future remnant liver. Transient elastography could not be performed in 22 cases due to tumor size. We defined the group with liver stiffness measurement (LSM) $\geq 7.9 \mathrm{kPa}$ as the high LSM group ( $\mathrm{n}=29,40 \%)$ and those with LSM $<7.9 \mathrm{kPa}$ as the low LSM group ( $=44,60 \%)$. We investigated the outcome after hepatectomy and the correlations between the VTQ of future remnant liver and other indicators for hepatic fibrosis.

Results: The high VTQ group showed significantly higher postoperative ascites (19\% vs. 3\%; $\mathrm{P}=0.01)$, pathological fibrosis ( $19 \%$ vs. $5 \%$; $\mathrm{P}=0.03)$, and rates of patients with postoperative T-bil $\geq 2.0 \mathrm{mg} / \mathrm{dL}$ (70\% vs. $40 \% ; \mathrm{P}<0.01)$. The high LSM group showed no significant postoperative outcomes compared to the low LSM group. The high VTQ group showed a higher frequency of male gender (78\% vs. 57\%; $\mathrm{P}=0.03)$, higher indocyanine green retention rate at $15 \mathrm{~min}$ (ICGR15) (10.5\% vs. 6.3\%; $\mathrm{P}<0.01$ ), hyaluronic acid (100 vs. $67 \mathrm{ng} / \mathrm{mL} ; \mathrm{P}=0.02)$, type $\mathrm{IV}$ collagen $7 \mathrm{~S}$ (7.6 vs. $5.1 \mathrm{ng} / \mathrm{mL} ; \mathrm{P}<0.01)$, Mac-2 binding protein glycan isomer (M2BPGi) (1.19 vs. 1.00; $\mathrm{P}=0.01)$, Fibrosis-4 (FIB-4) index (2.25 vs. 1.76; $\mathrm{P}=0.01$ ), and aspartate aminotransferase to platelet ratio index (APRI) score (0.64 vs. $0.41 ; \mathrm{P}<0.01)$. We also observed an especially strong positive correlation between the high VTQ and hyaluronic acid or type IV collagen 7S.

Conclusions: Elastography with VTQ for future remnant liver before major hepatectomy is an accurate and useful method as a preoperative evaluation.

Keywords: Elastography; virtual touch quantification (VTQ); future remnant liver (FRL); hepatectomy

Submitted Sep 18, 2020. Accepted for publication Feb 18, 2021.

doi: 10.21037/qims-20-1073

View this article at: http://dx.doi.org/10.21037/qims-20-1073

\footnotetext{
$\wedge$ ORCID: 0000-0002-5187-6753.
} 


\section{Introduction}

The complete resection of malignant hepatobiliary tumors is crucial for achieving long-term survival $(1,2)$. Major hepatectomy is often required for complete resection, and one of the main causes of unresectability is insufficient liver functional reserve or future remnant liver (FRL) volume. Hepatectomy should be performed without excess or deficiency within the safe limits of the resection volume determined by liver functional reserve. Although recent technological developments for hepatectomy and perioperative management have resulted in decreased mortality rates, hepatectomy is a highly invasive surgical procedure with a high morbidity rate in terms of gastroenterological surgery (3). According to the Japanese National Clinical Database of 14,970 cases with hepatectomy of more than 1 section, except for left lateral sectionectomy, rates of 90-day in-hospital mortality and overall morbidity were $3.7 \%$ and $25.7 \%$ (3). Posthepatectomy liver failure (PHLF), such as hyper bilirubinemia, the elevation of prothrombin time (PT), or international normalized ratio (INR) levels, and massive ascites were the main cause of death after hepatic resection, with incidences varying from $1.2 \%$ to $32 \%$ (4-6). To avoid PHLF, various liver functional reserve assessments were performed, such as platelet count, indocyanine green retention rate at $15 \mathrm{~min}$ (ICGR15), 99mTc-galactosyl human serum albumin (GSA) scintigraphy, hyaluronic acid, type IV collagen $7 \mathrm{~S}$, Mac-2 binding protein glycan isomer (M2BPGi), Fibrosis-4 (FIB-4) index, aspartate aminotransferase to platelet ratio index (APRI), ChildPugh classification, Albumin-Bilirubin (ALBI) grade, and Albumin-Indocyanine Green Evaluation (ALICE) grade.

The evaluation of liver stiffness by transient elastography has been reported as a useful modality for predictor PHLF $(7,8)$. Transient elastography measures the propagation velocity of a single-cycle shear wave generated by a probe (9), with values expressed in units of pressure (kilopascal, $\mathrm{kPa}$ ).

However, transient elastography could be performed for only the right hepatic lobe. Liver elastography with virtual touch quantification (VTQ) measures the velocity of shear wave generated by a short-duration acoustic force impulse, with values expressed in units of velocity $(\mathrm{m} / \mathrm{s})(10)$. VTQ can evaluate not only the right hepatic lobe but also the left hepatic lobe and has the advantage of real-time elastography. Thus, VTQ might be appropriate for the evaluation of FRL after right or left hepatectomy. There are some reports that VTQ was predictive factor for PHLF or useful for evaluating liver fibrosis $(11,12)$. However, these studies consisted of major and minor hepatectomies. There were no reports consisted of only major hepatectomy or evaluated FRL. Because the volume reduction of major resection is bigger than minor resection, PHLF in major resection tends to occur by volume and characteristics of remnant liver. Hence, the evaluation of VTQ in FRL of major resection is important and meaningful.

In this study, we compared the outcome after hepatectomy and the results of VTQ at FRL. We also investigated these outcomes regarding transient elastography. In addition, the relationships between the results of VTQ at FRL and other preoperative factors, the results of VTQ on resected side and pathological fibrosis were investigated.

\section{Methods}

\section{Patients}

Between August 2017 and May 2020, 95 patients underwent liver elastography with VTQ and both FRL and resected side before hepatectomy with more than two sections, except for central bisectionectomy at the Gastroenterological Surgery I unit of Hokkaido University Hospital in Sapporo, Japan. The diagnoses and type of hepatectomies were shown Table 1. The FIB-4 index and APRI were calculated as follows: FIB-4 index $=$ age (in years) $\times$ aspartate aminotransferase (AST) (U/L)]/[platelet count $\left(10^{9} / \mathrm{L}\right) \times$ alanine aminotransferase (ALT) $\left.(\mathrm{U} / \mathrm{L})^{1 / 2}\right](13)$, APRI $=($ AST/upper limit of normal $\times 100) /$ platelet count $\left(10^{9} / \mathrm{L}\right)(13)$.

This study was approved by the Hokkaido University Hospital Voluntary Clinical Study Committee (approval 17-0335) and was performed according to the Helsinki Declaration guidelines.

\section{Liver elastography}

VTQ was performed by using an ACUSON S2000 instrument (Siemens AG, Erlangen, Germany) with a 4 C ultrasound probe $(4.5 \mathrm{MHz})$. Values were expressed in meters per second ( $\mathrm{m} / \mathrm{s})$. According to the World Federation for Ultrasound in Medicine and Biology guidelines (14), VTQ measurements were performed under breath hold. VTQ was measured at two sites: one in the right lobe of the liver, accessed through the hypochondrium 
Table 1 Patient diagnoses and operative procedures

\begin{tabular}{lc}
\hline Variables & Value $(\mathrm{n}=95)$ \\
\hline Diagnosis & 39 \\
Hepatocellular carcinoma & 14 \\
Intrahepatic cholangiocarcinoma & 3 \\
Combined hepatocellular and & \\
cholangiocarcinoma & 10 \\
Bile duct cancer & 15 \\
Metastatic liver cancer from colorectal cancer & 8 \\
Alveolar echinococcosis & 6 \\
Others & \\
Operative procedure & 52 \\
Right hepatectomy & 7 \\
Right trisegmentectomy & 33 \\
Left hepatectomy & 3 \\
Left trisegmentectomy & \\
\hline
\end{tabular}

space (Figure 1A), and the other in the left lobe of the liver, accessed through the overlying intercostal space at a depth of 2-4 cm, as described previously (Figure 1B) (15). The area of region of interest (ROI) of VTQ is $0.6 \mathrm{~cm} \times 1.0 \mathrm{~cm}$. Eight registered sonographers measured VTQ. Inter- and intra-operator reliabilities were good in previous report at our institution which showed that interobserver agreement was analyzed using the intraclass correlation coefficient for liver stiffness of 0.70 (95\% confidence interval: $0.44-0.85$, $\mathrm{P}<0.01)$ among the patients measured liver stiffness using VTQ in 28 healthy volunteers (27.5 \pm 8.3 years, 14 male) (16). To ensure the accuracy, success rate more than $60 \%$ and the interquartile range and all validated measurements was within $30 \%$ of the median value were used as valid VTQ value. The median value was considered representative of the VTQ measurement only if the interquartile range of all validated measurements was within $30 \%$ of the median value.

VTQ of FRL indicated VTQ of the left lobe when right lobectomy was performed and VTQ of the right lobe when left lobectomy was performed. We defined the group with VTQ of FRL $\geq 1.52 \mathrm{~m} / \mathrm{s}$ as the high VTQ group $(\mathrm{n}=37,39 \%)$ and those with VTQ of FRL $<1.52 \mathrm{~m} / \mathrm{s}$ as the low VTQ group $(\mathrm{n}=58,61 \%)$ using a receiver operating characteristic (ROC) curve for the presence of postoperative ascites. In this cohort, the cases which showed PHLF
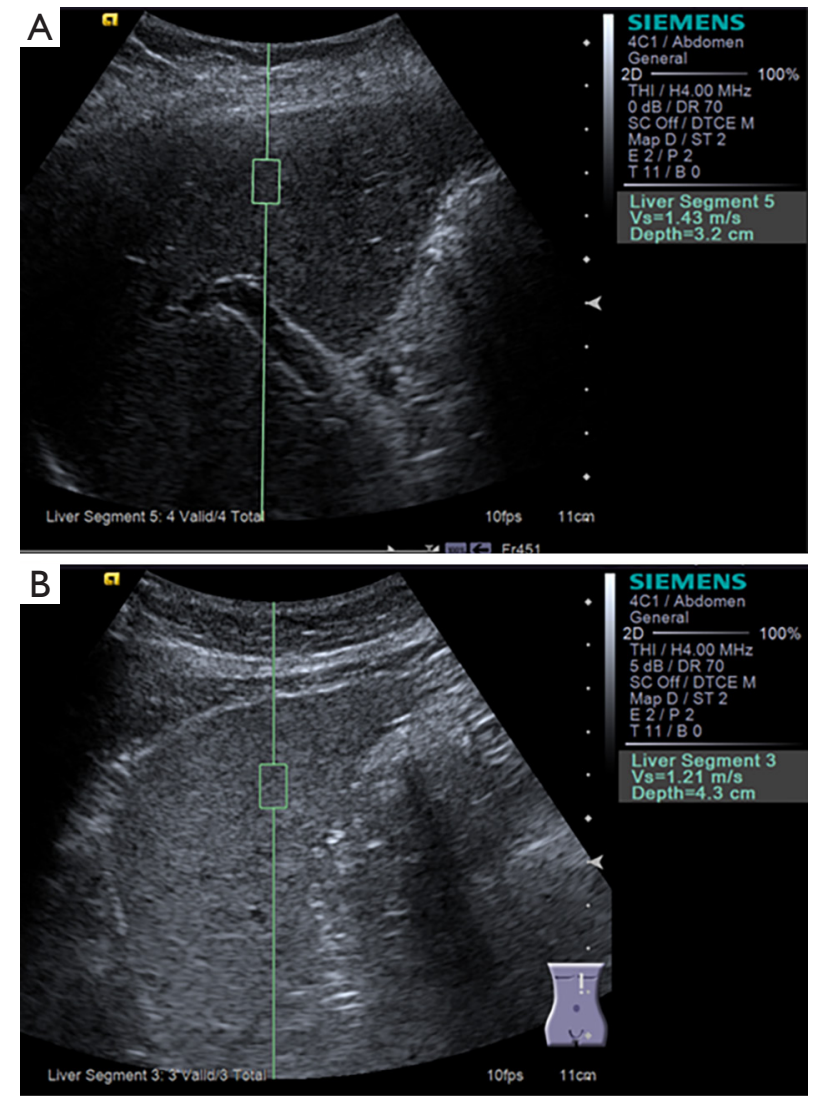

Figure 1 VTQ measurements. (A) VTQ was evaluated in the hepatic right lobe. (B) VTQ was evaluated in the hepatic left lobe. VTQ, virtual touch quantification.

according to International Study Group of Liver Surgery (ISGLS) criteria were only three cases (4). Therefore, we chose ascites after hepatectomy as cut-off point. Area under ROC curve (AUC) value for VTQ was 0.6854 (Figure 2A).

In addition, we calculated modified VTQ (mVTQ) adjusted with the effective liver resection rates (ELRRs). ELRRs was defined as (resection volume - tumor volume)/ (whole liver volume - tumor volume) $\times 100$. $\mathrm{mVTQ}$ was calculated as VTQ $\times$ ELRRs/100. We defined the group with $\mathrm{mVTQ} \geq 0.67 \mathrm{~m} / \mathrm{s}$ as the high $\mathrm{mVTQ}$ group $(\mathrm{n}=40$, $42 \%$ ) and those with $\mathrm{mVTQ}<0.67 \mathrm{~m} / \mathrm{s}$ as the low VTQ group $(\mathrm{n}=55,58 \%)$ using a ROC curve for the presence of postoperative ascites. AUC value for mVTQ was 0.7132 (Figure 2B).

Transient elastography was performed by using FibroScan 502 (Echosens, Paris, France). Liver stiffness measurement (LSM) was measured with the M-probe. As described previously, the examinations were performed 

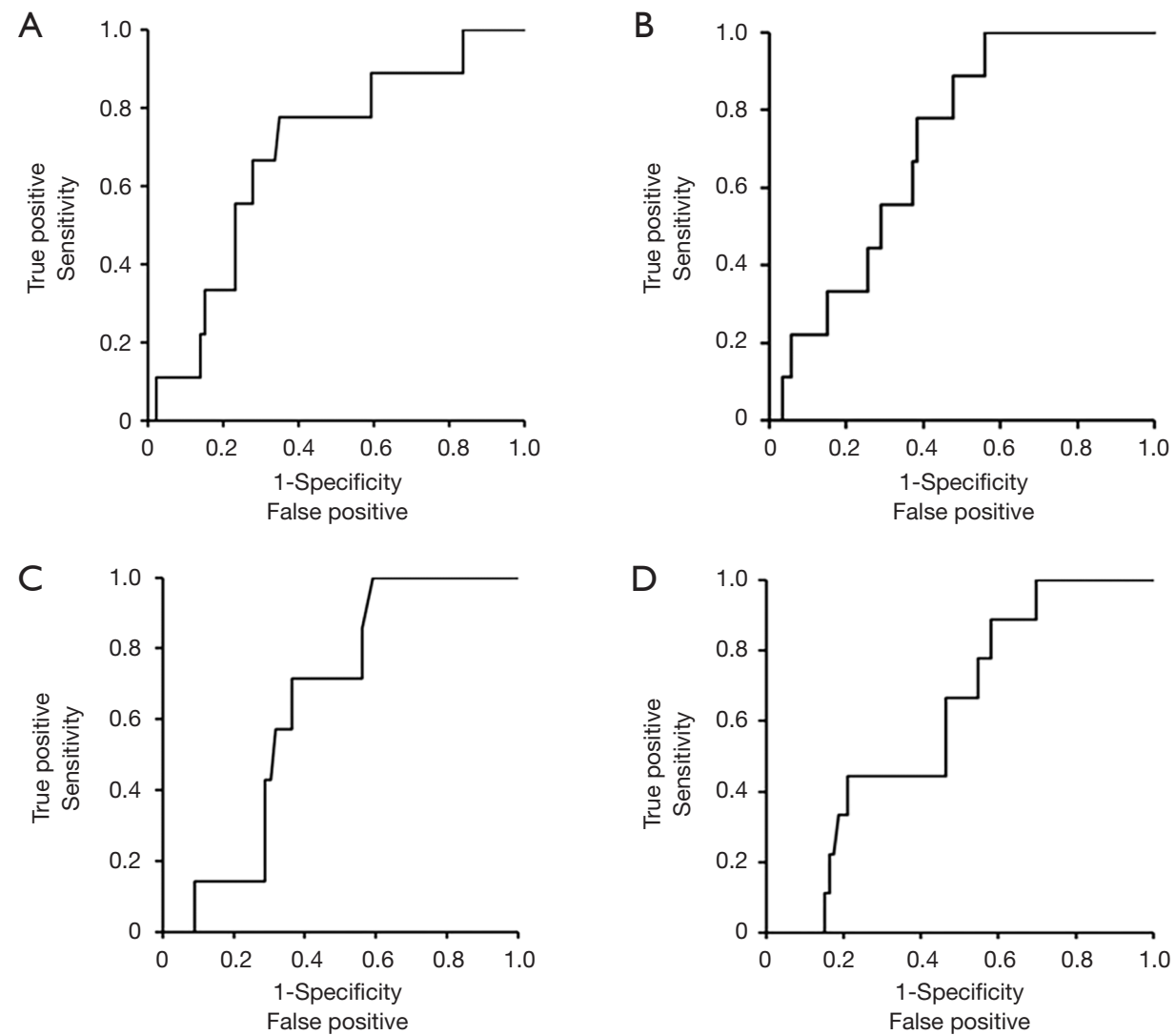

Figure 2 ROC curve between covariates and postoperative ascites. (A) Receiver operating characteristic (ROC) curve between VTQ and the presence of postoperative ascites according to Clavien-Dindo classification grades III-V. Area under ROC curve (AUC) value was 0.6854. (B) ROC curve between $\mathrm{mVTQ}$ and the presence of postoperative ascites according to Clavien-Dindo classification grades III-V. AUC value was 0.7132. (C) ROC curve between LSM and the presence of postoperative ascites according to Clavien-Dindo classification grades III-V. AUC value was 0.6461. (D) ROC curve between ELRRs and the presence of postoperative ascites according to Clavien-Dindo classification grades III-V. AUC value was 0.6156. VTQ, virtual touch quantification; LSM, liver stiffness measurement.

with at least 10 valid measurements with a right intercostal scanning. Effective measurements were defined as those $>60 \%$ with an interquartile range of $<30 \%$ (17).

Transient elastography could not be performed in 22 cases due to tumor size. We defined the group with LSM $\geq 7.9 \mathrm{kPa}$ as the high LSM group ( $\mathrm{n}=29,40 \%)$ and the group with LSM $<7.9 \mathrm{kPa}$ as the low LSM group ( $\mathrm{n}=44,60 \%)$ using a ROC curve for the presence of postoperative ascites. AUC value for LSM was 0.6461 (Figure 2C).

\section{Hepatectomy}

Our criteria specify that patients with uncontrollable ascites and patients with serum total bilirubin (T-bil) levels of $2.0 \mathrm{mg} / \mathrm{dL}$ or more are not indicated for hepatectomy, and the surgical procedure was determined according to the ICGR15 (18). We determined the liver resection volume according to the ICGR15 and ELRRs and FRL volume by computed tomography (CT)-volumetry. We performed hemi- or extended lobectomy for patients whose ICGR15 was $15 \%$ or less. If the cases in which ELRRs were more than $60 \%$ or the FRL volume was less than $400 \mathrm{~mL}$, we considered portal vein embolization.

We evaluated a ROC curve between ELRRs and the presence of postoperative ascites. Cut-off point and AUC value were $46.8 \%, 0.6156$, respectively (Figure $2 D$ ).

\section{Statistical analysis}

Univariate analyses were performed using the Mann- 
Table 2 Clinicopathological characteristics and outcomes in this cohort

\begin{tabular}{|c|c|}
\hline Characteristics & Value $(n=95)$ \\
\hline \multicolumn{2}{|l|}{ Epidemiology } \\
\hline Median age (years) & $68(17-84)$ \\
\hline Sex (male/female) & $62 / 33$ \\
\hline HBs-Ag positive (\%) & 8 \\
\hline HCV-Ab positive (\%) & 5 \\
\hline $\mathrm{BMI}\left(\mathrm{kg} / \mathrm{m}^{2}\right)$ & $22.9(15.1-39.9)$ \\
\hline Long history of drinking (\%) & 4 \\
\hline \multicolumn{2}{|l|}{ Biochemical factors } \\
\hline PIt $\left(\times 10^{4} / \mu \mathrm{L}\right)$ & $24.1(10.9-90.9)$ \\
\hline PT-INR & $0.99(0.84-1.26)$ \\
\hline Alb (g/dL) & $4.0(3.0-4.8)$ \\
\hline ICGR15 (\%) & $8.4(1.4-28.1)$ \\
\hline \multicolumn{2}{|l|}{ 99mTc-GSA scintigraphy } \\
\hline LHL15 & $0.929(0.686-0.972)$ \\
\hline \multicolumn{2}{|l|}{ Other indicators regarding fibrosis } \\
\hline $\mathrm{HA}(\mathrm{ng} / \mathrm{mL})$ & $73.5(10.0-811.0)$ \\
\hline IV collagen (ng/mL) & $5.5(2.8-14.4)$ \\
\hline M2BPGi & $0.99(0.33-5.40)$ \\
\hline FIB-4 index & $1.95(0.12-5.87)$ \\
\hline APRI & $0.49(0.14-3.08)$ \\
\hline Pathological liver fibrosis ( $¥ 3$ or 4) (\%) & 11 \\
\hline \multicolumn{2}{|l|}{ Hepatic elastography } \\
\hline Median VTQ of FRL (m/s) & $1.38(0.72-3.38)$ \\
\hline Median VTQ of future resected lobe $(\mathrm{m} / \mathrm{s})$ & $1.37(0.88-4.11)$ \\
\hline Median LSM (kPa) & $6.5(2.8-72.0)$ \\
\hline
\end{tabular}

Table 2 (continued)

Whitney $U$ test for continuous variables and the chi-square test for noncontinuous variables. Multivariate analyses were performed using logistic regression model analyses. Pearson's correlation coefficients were used to analyze the correlation between the VTQ of FRL and hyaluronic acid, type IV collagen 7S, M2BPGi, FIB-4 index, APRI, and ICGR15. This analysis was also performed between the VTQ of the future resected lobe before hepatectomy and histological liver fibrosis of the resected liver. A P value of $<0.05$ was considered significant. Statistical analyses
Table 2 (continued)

\begin{tabular}{|c|c|}
\hline Characteristics & Value $(n=95)$ \\
\hline \multicolumn{2}{|l|}{ Surgical factors } \\
\hline Using Pringle maneuver (\%) & 86 \\
\hline Median ELRRs (\%) & $46.2(4.9-62.1)$ \\
\hline Median operative time (min) & $316(155-788)$ \\
\hline Median blood loss (mL) & $310(0-3,270)$ \\
\hline \multicolumn{2}{|l|}{ Posthepatectomy outcomes } \\
\hline Mortality rates (\%) & 0 \\
\hline Ascites* (\%) & 9 \\
\hline Maximum T-bil $\geq 2.0 \mathrm{mg} / \mathrm{dL}(\%)$ & 52 \\
\hline Maximum PT-INR $\geq 1.2(\%)$ & 76 \\
\hline Median postoperative stay (days) & $13(6-130)$ \\
\hline \multicolumn{2}{|c|}{$\begin{array}{l}\text { *, postoperative ascites according to Clavien-Dindo classification } \\
\text { grades III-V. HBs-Ag, hepatitis B surface antigen; HCV-Ab, } \\
\text { hepatitis C virus antibody; BMI, body mass index; PIt, platelet; } \\
\text { PT-INR, prothrombin time international normalized ratio; Alb, } \\
\text { albumin; ICGR15, indocyanine green retention rate at } 15 \text { min; } \\
\text { 99mTc-GSA, technetium 99m diethylenetriaminepentaacetic } \\
\text { acid-galactosyl-human serum albumin; LHL15, receptor } \\
\text { index: uptake ratio of the liver to that of the liver plus heart } \\
\text { at } 15 \text { min of technetium 99m diethylenetriaminepentaacetic } \\
\text { acid-galactosyl-human serum albumin scintigraphy; HA, } \\
\text { hyaluronic acid; IV collagen, type IV collagen 7S; M2BPGi, } \\
\text { Mac-2 binding protein glycan isomer; FIB-4, Fibrosis-4; APRI, } \\
\text { aspartate aminotransferase to platelet ratio index; f3, bridging } \\
\text { fibrosis; f4, cirrhosis; VTQ, virtual touch quantification; FRL, } \\
\text { future remnant liver; LSM, liver stiffness measurement; ELRRs, } \\
\text { effective liver resection rates; T-bil, total bilirubin. }\end{array}$} \\
\hline
\end{tabular}

were performed using JMP Pro 14.0.0 for Windows (SAS Institute Inc., NC, USA).

\section{Results}

\section{Perioperative data of patients}

Patient perioperative data are provided in Table 2. The median VTQ of FRL was $1.38(0.72-3.38) \mathrm{m} / \mathrm{s}$. The mortality rate was $0 \%$. The rate of postoperative ascites according to Clavien-Dindo classification grades III-V was $9 \%$. The median postoperative hospital stay was 13 (6-130) days. PHLF according to the ISGLS (4) occurred in 3 patients (3.2\%). Two patients $(2.1 \%)$ were categorized as grade $\mathrm{A}, 1$ patient $(1.1 \%)$ as grade $\mathrm{B}$, and no patients as grade $\mathrm{C}$. 


\section{VTQ of FRL and the outcome after bepatectomy}

The clinicopathological characteristics and outcomes after hepatectomy between the high and low-VTQ groups are provided in Table 3. The following variables were significantly different between these two groups: male gender ( $78 \%$ vs. $57 \%$; $\mathrm{P}=0.03)$, ICGR 15 (10.5\% vs. $6.3 \%$; $\mathrm{P}<0.01)$, hyaluronic acid (100 vs. $67 \mathrm{ng} / \mathrm{mL} ; \mathrm{P}=0.02)$, type IV collagen $7 \mathrm{~S}$ (7.6 vs. $5.1 \mathrm{ng} / \mathrm{mL} ; \mathrm{P}<0.01)$, M2BPGi (1.19 vs. 1.00; $\mathrm{P}=0.01)$, FIB-4 index (2.25 vs. 1.76; $\mathrm{P}=0.01)$, and APRI score $(0.64 v s .0 .41 ; \mathrm{P}<0.01)$.

The high VTQ group showed significantly higher postoperative ascites $(19 \% v s .3 \% ; \mathrm{P}=0.01)$, pathological fibrosis ( $\mathrm{f} 3$ or 4$)(19 \%$ vs. $5 \%$; $\mathrm{P}=0.03)$, and the rate of patients with T-bil $\geq 2.0 \mathrm{mg} / \mathrm{dL}$ ( $70 \%$ vs. $40 \% ; \mathrm{P}<0.01)$. The rates of patients with PT-INR $\geq 1.2$ and postoperative stay were not significantly different.

The sensitivity, specificity, accuracy, positive predictive value, and negative predictive value for predicting the ascites according to Clavien-Dindo classification grades III$\mathrm{V}$ after hepatectomy were $77.8 \%, 65.1 \%, 66.3 \%, 18.9 \%$, $96.6 \%$, respectively.

Median VTQ of the left and right lobes were 1.55 (0.852.70), $1.14(0.72-3.38) \mathrm{m} / \mathrm{s}$, respectively $(\mathrm{P}<0.01)$.

\section{$m V T Q$ and the outcome after hepatectomy}

The clinicopathological characteristics and outcomes after hepatectomy between the high and low-mVTQ groups are provided in Table 4. The following variables were significantly different between these two groups: type IV collagen $7 \mathrm{~S}(7.2$ vs. $5.2 \mathrm{ng} / \mathrm{mL} ; \mathrm{P}<0.01)$, and APRI score (0.56 vs. $0.43 ; \mathrm{P}=0.02$ ).

The high $\mathrm{mVTQ}$ group showed significantly higher postoperative ascites $(20 \%$ vs. $2 \%$; $\mathrm{P}<0.01)$, the rate of patients with T-bil $\geq 2.0 \mathrm{mg} / \mathrm{dL}$ (75\% vs. $35 \% ; \mathrm{P}<0.01)$, and those with PT-INR $\geq 1.2$ ( $88 \%$ vs. $67 \% ; \mathrm{P}=0.02$ ). The rates of patients and postoperative stay were not significantly different.

The sensitivity, specificity, accuracy, positive predictive value, and negative predictive value for predicting the ascites according to Clavien-Dindo classification grades III$\mathrm{V}$ after hepatectomy were $88.9 \%, 62.8 \%, 65.3 \%, 20.0 \%$, $98.2 \%$, respectively. mVTQ concerning postoperative ascites showed higher AUC value 0.7132 compared to VTQ, transient elastography, and ELRRs (0.6854, 0.6461, and 0.6156$)$.
Transient elastography and the outcome after hepatectomy

LSM was evaluated by using transient elastography. The clinicopathological characteristics and outcome after hepatectomy between the high- and low-LSM groups are provided in Table 5. The following variables were significantly different between these two groups: PTINR (1.00 vs. 0.96; $\mathrm{P}<0.01)$, ICGR15 (10.1\% vs. 7.6\%; $\mathrm{P}=0.02)$, hyaluronic acid (100 vs. $60 \mathrm{ng} / \mathrm{mL} ; \mathrm{P}<0.01)$, type IV collagen 7S (6.9 vs. $4.9 \mathrm{ng} / \mathrm{mL} ; \mathrm{P}<0.01)$, M2BPGi (1.30 vs. $0.75 ; \mathrm{P}<0.01)$, APRI score $(0.65$ vs. $0.39 ; \mathrm{P}<0.01)$, and pathological fibrosis ( $\mathrm{f} 3$ or 4$)(21 \%$ vs. $2 \%$; $\mathrm{P}<0.01)$. However, these two groups showed no significant differences regarding postoperative outcome.

\section{Correlations between the VTQ of FRL and ICGR15, byaluronic acid, type IV collagen 7S, M2BPGi, FIB-4 index, and APRI}

Table 6 and Figure 3 showed the correlation coefficients between VTQ of FRL and ICGR15, hyaluronic acid, type IV collagen 7S, M2BPGi, FIB-4 index, and APRI. In particular, VTQ of FRL showed strong correlation to hyaluronic acid and type IV collagen $7 \mathrm{~S}$ (correlation coefficients; 0.3266 and 0.4903 , respectively).

\section{Correlation between the prediction of fibrosis score by $V T Q$ of a future resected lobe and actual histological liver fibrosis of the resected liver}

The correlation coefficient between the prediction of fibrosis score by VTQ of a future resected side and actual histological liver fibrosis of the resected liver was 0.2446 .

\section{Discussion}

We investigated the outcome after hepatectomy and the results of VTQ at FRL or transient elastography, and the relationships between the results of VTQ at FRL and other preoperative factors regarding hepatic fibrosis. The high VTQ group showed significantly higher postoperative ascites and rates of patients with T-bil $\geq 2.0 \mathrm{mg} / \mathrm{dL}$ and those with PT-INR $\geq 1.2$. Meanwhile, the high LSM group showed no significant postoperative outcomes compared to the low LSM group. Regarding clinicopathological characteristics, the high VTQ group showed a higher frequency of male gender, pathological fibrosis (f3 or 4 ) 
Table 3 Clinicopathological characteristics and outcomes between high and low VTQ groups

\begin{tabular}{|c|c|c|c|}
\hline Characteristics & High VTQ $(n=37)$ & Low VTQ (n=58) & $\mathrm{P}$ \\
\hline Median age (years) & $68(44-79)$ & $68(17-84)$ & 0.83 \\
\hline Sex (male/female) & $29 / 8$ & $33 / 25$ & 0.03 \\
\hline HBs-Ag positive (\%) & 11 & 7 & 0.50 \\
\hline BMI $\left(k g / m^{2}\right)$ & $23.9(15.1-39.9)$ & $22.4(15.8-31.2)$ & 0.16 \\
\hline Long history of drinking (\%) & 5 & 3 & 0.64 \\
\hline \multicolumn{4}{|l|}{ Biochemical factors } \\
\hline Plt $\left(\times 10^{4} / \mu \mathrm{L}\right)$ & $23.4(13.4-55.6)$ & $24.2(10.9-90.9)$ & 0.71 \\
\hline \multicolumn{4}{|l|}{ 99mTc-GSA scintigraphy } \\
\hline LHL15 & $0.930(0.686-0.972)$ & $0.929(0.789-0.965)$ & 0.94 \\
\hline \multicolumn{4}{|l|}{ Other indicators regarding fibrosis } \\
\hline $\mathrm{HA}(\mathrm{ng} / \mathrm{mL})$ & $109(33-811)$ & $65(10-549)$ & $<0.01$ \\
\hline IV collagen (ng/mL) & $7.7(4.0-14.4)$ & $5.1(2.8-11.2)$ & $<0.01$ \\
\hline M2BPGi & $0.98(0.36-3.81)$ & $0.85(0.33-3.66)$ & 0.25 \\
\hline FIB-4 index & $2.23(0.64-5.47)$ & $1.78(0.12-5.87)$ & 0.04 \\
\hline Median operative time (min) & $328(210-788)$ & $304(155-786)$ & 0.24 \\
\hline Median blood loss (mL) & $380(40-3,270)$ & $268(0-1,295)$ & $<0.01$ \\
\hline \multicolumn{4}{|l|}{ Posthepatectomy outcomes } \\
\hline Mortality rates (\%) & 0 & 0 & - \\
\hline Ascites $^{*}(\%)$ & 19 & 3 & 0.01 \\
\hline Maximum T-bil $\geq 2.0$ mg/dL (\%) & 70 & 40 & $<0.01$ \\
\hline Maximum PT-INR $\geq 1.2$ (\%) & 84 & 71 & 0.14 \\
\hline Median postoperative stay (days) & $13(8-130)$ & $12(6-70)$ & 0.29 \\
\hline
\end{tabular}

*, postoperative ascites according to Clavien-Dindo classification grades III-V. VTQ, virtual touch quantification; HBs-Ag, hepatitis B surface antigen; HCV-Ab, hepatitis C virus antibody; BMI, body mass index; PIt, platelet; PT-INR, prothrombin time international normalized ratio; Alb, albumin; ICGR15, indocyanine green retention rate at $15 \mathrm{~min}$; 99mTc-GSA, technetium 99m diethylenetriaminepentaacetic acid-galactosyl-human serum albumin; LHL15, receptor index: uptake ratio of the liver to that of the liver plus heart at 15 min of technetium 99m diethylenetriaminepentaacetic acid-galactosyl-human serum albumin scintigraphy; HA, hyaluronic acid; IV collagen, type IV collagen 7S; M2BPGi, Mac-2 binding protein glycan isomer; APRI, aspartate aminotransferase to platelet ratio index; f3, bridging fibrosis; f4, cirrhosis; ELRRs, effective liver resection rates; T-bil, total bilirubin. 
Table 4 Clinicopathological characteristics and outcomes between high and low mVTQ groups

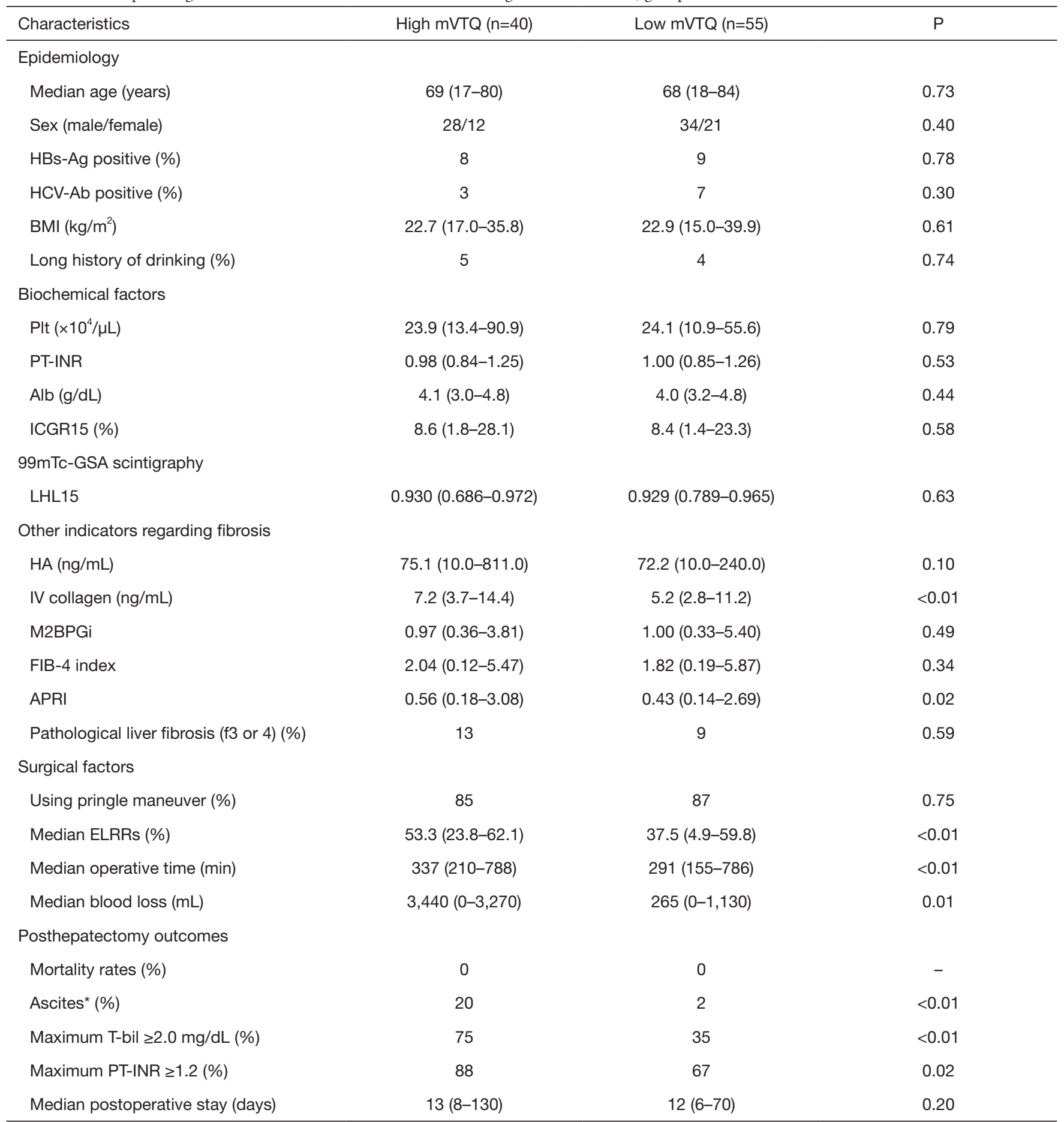

*, postoperative ascites according to Clavien-Dindo classification grades III-V. mVTQ, modified virtual touch quantification; HBs-Ag, hepatitis B surface antigen; HCV-Ab, hepatitis C virus antibody; BMI, body mass index; PIt, platelet; PT-INR, prothrombin time international normalized ratio; Alb, albumin; ICGR15, indocyanine green retention rate at 15 min; 99mTc-GSA, technetium 99m diethylenetriaminepentaacetic acid-galactosyl-human serum albumin; LHL15, receptor index: uptake ratio of the liver to that of the liver plus heart at 15 min of technetium 99m diethylenetriaminepentaacetic acid-galactosyl-human serum albumin scintigraphy; HA, hyaluronic acid; IV collagen, type IV collagen 7S; M2BPGi, Mac-2 binding protein glycan isomer; FIB-4, Fibrosis-4; APRI, aspartate aminotransferase to platelet ratio index; $\mathrm{f} 3$, bridging fibrosis; $\mathrm{f} 4$, cirrhosis; ELRRs, effective liver resection rates; T-bil, total bilirubin. 
Table 5 Clinicopathological characteristics and outcomes between high and low LSM groups

\begin{tabular}{|c|c|c|c|}
\hline Characteristics & High LSM $(n=29)$ & Low LSM $(n=44)$ & $\mathrm{P}$ \\
\hline Median age (years) & $68(54-79)$ & $68(18-84)$ & 0.58 \\
\hline Sex (male/female) & $21 / 8$ & $26 / 18$ & 0.24 \\
\hline HBs-Ag positive (\%) & 14 & 7 & 0.32 \\
\hline BMI $\left(\mathrm{kg} / \mathrm{m}^{2}\right)$ & $21.9(15.1-39.9)$ & $23.6(15.8-31.2)$ & 0.40 \\
\hline Long history of drinking (\%) & 7 & 0 & 0.07 \\
\hline \multicolumn{4}{|l|}{ Biochemical factors } \\
\hline PIt $\left(\times 10^{4} / \mu \mathrm{L}\right)$ & $23.9(12.4-55.6)$ & $23.9(10.9-46.7)$ & 0.87 \\
\hline \multicolumn{4}{|l|}{ 99mTc-GSA scintigraphy } \\
\hline LHL15 & $0.923(0.716-0.957)$ & $0.929(0.828-0.972)$ & 0.30 \\
\hline \multicolumn{4}{|l|}{ Other indicators regarding fibrosis } \\
\hline $\mathrm{HA}(\mathrm{ng} / \mathrm{mL})$ & $100(40-811)$ & $60(10-549)$ & $<0.01$ \\
\hline IV collagen (ng/mL) & $6.9(4.3-14.4)$ & $4.9(2.8-11.2)$ & $<0.01$ \\
\hline M2BPGi & $1.28(0.46-3.81)$ & $0.75(0.33-3.43)$ & $<0.01$ \\
\hline FIB-4 index & $2.14(0.64-5.87)$ & $1.68(0.19-3.60)$ & 0.06 \\
\hline Median operative time (min) & $345(211-788)$ & $301(155-786)$ & 0.13 \\
\hline Median blood loss (mL) & $290(85-2,000)$ & $255(0-1,295)$ & 0.06 \\
\hline \multicolumn{4}{|l|}{ Posthepatectomy outcomes } \\
\hline Mortality rates (\%) & 0 & 0 & - \\
\hline Ascites $^{*}(\%)$ & 17 & 5 & 0.07 \\
\hline Maximum T-bil $\geq 2.0$ mg/dL (\%) & 66 & 45 & 0.09 \\
\hline Maximum PT-INR $\geq 1.2(\%)$ & 90 & 70 & 0.05 \\
\hline Median postoperative stay (days) & $15(7-130)$ & $12(6-70)$ & 0.10 \\
\hline
\end{tabular}

*, postoperative ascites according to Clavien-Dindo classification grades III-V. LSM, liver stiffness measurement; HBs-Ag, hepatitis B surface antigen; HCV-Ab, hepatitis C virus antibody; BMI, body mass index; PIt, platelet; PT-INR, prothrombin time international normalized ratio; Alb, albumin; ICGR15, indocyanine green retention test at $15 \mathrm{~min}$; 99mTc-GSA, technetium 99m diethylenetriaminepentaacetic acid-galactosyl-human serum albumin; LHL15, receptor index: uptake ratio of the liver to that of the liver plus heart at 15 min of technetium 99m diethylenetriaminepentaacetic acid-galactosyl-human serum albumin scintigraphy; HA, hyaluronic acid; IV collagen, type IV collagen 7S; M2BPGi, Mac-2 binding protein glycan isomer; APRI, aspartate aminotransferase to platelet ratio index; f3, bridging fibrosis; $\mathrm{f} 4$, cirrhosis; ELRRs, effective liver resection rates; T-bil, total bilirubin. 
Table 6 Correlation between the VTQ of FRL and ICGR15, HA, IV collagen, M2BPGi, FIB-4 index, APRI

\begin{tabular}{lc}
\hline Factors & Correlation coefficients \\
\hline ICGR15 & 0.1851 \\
HA & 0.3266 \\
IV collagen & 0.4903 \\
M2BPGi & 0.2049 \\
FIB-4 index & 0.1918 \\
APRI & 0.2429 \\
\hline
\end{tabular}

VTQ, virtual touch quantification; FRL, future remnant liver; ICGR15, indocyanine green retention test at $15 \mathrm{~min}$; HA, hyaluronic acid; IV collagen, type IV collagen 7S; M2BPGi, Mac-2 binding protein glycan isomer; APRI, aspartate aminotransferase to platelet ratio index. and higher ICGR15, hyaluronic acid, type IV collagen 7S, M2BPGi, FIB-4 index, and APRI score. We also observed an especially strong positive correlation between the high VTQ at FRL and hyaluronic acid or type IV collagen 7S. In addition, mVTQ concerning postoperative ascites showed higher AUC value 0.7132 compared to VTQ, LSM, and ELRRs (0.6854, 0.6461, and 0.6156).

Liver damage and liver fibrosis were known as a risk factor for PHLF or morbidities after hepatectomy (19). VTQ and transient elastography were methods as evaluation for liver damage and liver fibrosis. Our study showed that the rates of pathological liver fibrosis were significantly higher in high value group by both VTQ and transient elastography methods.

In the clinic, ultrasound strain elastography techniques
A

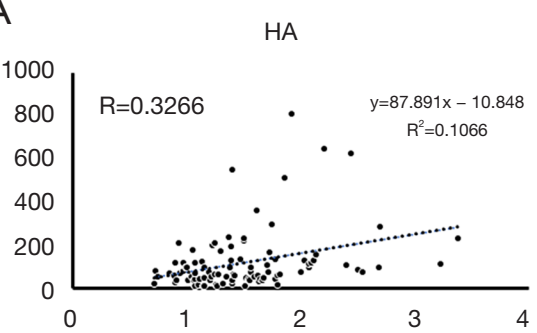

B

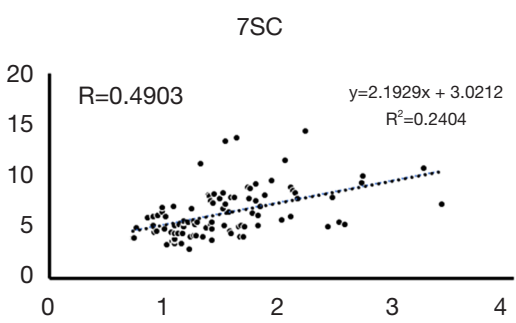

C

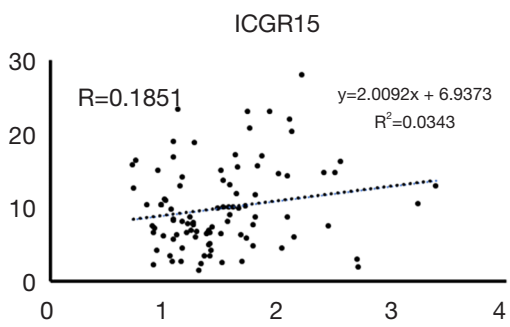

D

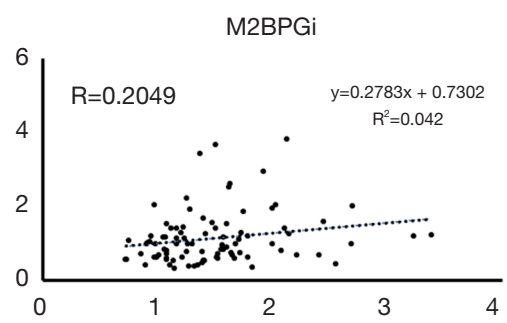

E

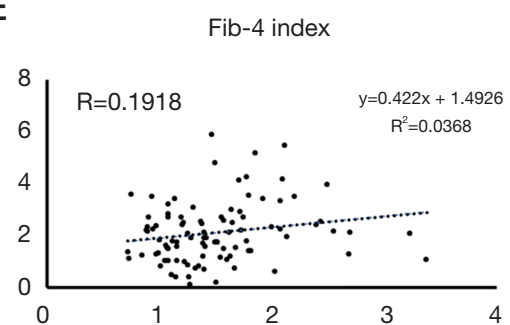

$\mathrm{F}$
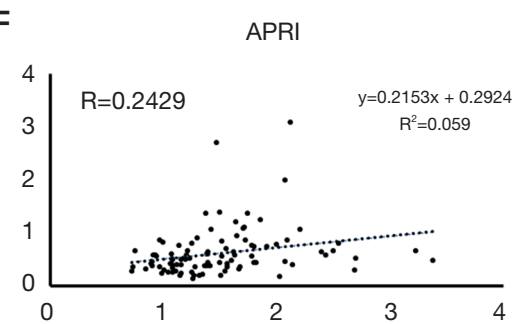

Figure 3 Correlation between VTQ and other markers of liver fibrosis. (A) Correlation between the virtual touch quantification (VTQ) of future remnant liver and hyaluronic acid (HA). The correlation coefficient was 0.3266. (B) Correlation between the VTQ of future remnant liver and type IV collagen 7S (7SC). The correlation coefficient was 0.4903. (C) Correlation between the VTQ of future remnant liver and indocyanine green retention rate at $15 \mathrm{~min}$ (ICGR15). The correlation coefficient was 0.1851. (D) Correlation between the VTQ of future remnant liver and Mac-2 binding protein glycan isomer (M2BPGi). The correlation coefficient was 0.2049. (E) Correlation between the VTQ of future remnant liver and Fibrosis-4 (FIB-4) index. The correlation coefficient was 0.1918. (F) Correlation between the VTQ of future remnant liver and aspartate aminotransferase to platelet ratio index (APRI). The correlation coefficient was 0.2429. 
were applied for the breast in 1993 (20) and for the prostate (21). However, these techniques were not suitable for organs such as the liver that move during respiration. Sandrin et al. reported that transient elastography quantified hepatic fibrosis and cirrhosis in a cohort of patients with HCV in 2003 (22). The mechanism of transient elastography involves vibrations of mild amplitude and low frequency that are transmitted by the transducer, inducing an elastic shear wave that propagates through the underlying tissues. Pulse-echo acquisitions are used to follow the propagation of the shear wave and to measure its velocity, which is related directly to tissue stiffness (9). Cescon et al. reported that elevated liver stiffness was an independent predictor of postoperative liver failure with transient elastography (7). However, some cases were inappropriate for transient elastography. Transient elastography cannot be applied to patients with ascites, narrow intercostal spaces, and overweight status.

VTQ is a point shear wave elastography (pSWE) and an acoustic radiation force impulse (ARFI) quantification that is employed as a novel technique (23). VTQ uses the acoustic push pulse to measure shear wave velocity $(\mathrm{m} / \mathrm{s})$ in a regional average and calculates the shear wave velocity within the ROI by measuring the time to peak displacement at each lateral location (24). This technique is accurate for even overweight and obese patients and can evaluate the left hepatic lobe (25). Nishio et al. reported that VTQ was useful for the prediction of PHLF and the estimation of the safe remnant liver volume rate range (11). Their study cohort showed that PHLF occurred in $21.5 \%$, with grade A in $9.6 \%$, grade B in $18.5 \%$, and grade C in $3.4 \%$. Meanwhile, the incidence rates in our study cohort were $3.2 \%, 2.1 \%, 1.1 \%$, and $0 \%$, respectively. Therefore, we could not show the impact of VTQ on PHLF. In general, the case with preoperative ascites was not adequate for surgery. It has been already reported that postoperative uncontrolled ascites was closely related to PHLF (26). Nanashima et al. reported that VTQ elastography was useful in evaluating impaired liver function before hepatectomy and predicting posthepatectomy morbidity or uncontrolled ascites (12). Han et al. also reported that the patients with SWE values higher than or equal to $6.9 \mathrm{kPa}$ had higher risk of PHLF, of which the sensitivity was $77.8 \%$ and the specificity was $78.0 \%$ (27). Therefore, VTQ was a useful method for predicting the outcome after hepatectomy. These studies included several types of hepatectomy, such as partial resection, sectionectomy, segmentectomy, and hemihepatectomy. Our study consists of only major hepatectomy, such as hemihepatectomy or trisegmentectomy. This study is the first study that evaluated FRL and focused on the cohort was only major hepatectomy.

In this study, we also investigated the impact of transient elastography on the outcome after hepatectomy. VTQ elastography was superior to transient elastography to predict the outcome after hepatectomy. Transient elastography is not good at evaluating the left hepatic lobe, whereas VTQ can evaluate the left hepatic lobe. The reasons for different outcomes between groups of VTQ and LSM might be due to the ability which could evaluate obese patients or FRL side. For the right side hepatectomy, the results of transient elastography might be affected by the tumor, the results of evaluation of FRL by VTQ might not.

Surgeons often evaluate the preoperative estimation of remnant hepatic function as a more precise preoperative evaluation by using combined $99 \mathrm{~m}$ Tc-GSA scintigraphy and CT-volumetry (28). It was known that the degree of hepatic fibrosis by liver biopsy was also different between the right and left lobes (29). Moreover, it was reported that the VTQ value of the left lobe tended to be higher than that of the right lobe (30). Our results showed also VTQ value was significantly higher in the left lobe than those in the right lobe. The precise mechanisms of this phenomenon are still elusive, but there are several possible contributing factors, such as the anatomical features of the left lobe surrounded by the diaphragm, stomach, and aorta, which might be influenced by respiratory fluctuations, the presence of food in the stomach, and the pulsation of the aorta (30). However, this difference might be crucially important for an accurate evaluation to determine the remnant side after hepatectomy. Moreover, mVTQ with consideration of both VTQ and ELRRs was useful indicator for predicting postoperative ascites in this study because liver resection rates is one of the major factors for PHLF (31).

We evaluated the correlation between the results of VTQ at FRL and the other preoperative characteristic factors that represent liver function, such as ICGR15, hyaluronic acid, type IV collagen 7S, M2BPGi, FIB-4 index, and APRI. The correlations with FIB-4 index or APRI were relatively weak compared to the other factors. Both FIB-4 index and APRI were calculated using AST or ALT. The cases with large tumor often show the elevation of AST/ALT (32). Therefore, in particular, FIB-4 index and APRI might be higher in the cases with large tumors than those of actual value.

The limitation of this study is the follows; first, our study cohort had very few cases with PHLF. Therefore, it was 
difficult to investigate whether VTQ elastography could predict PHLF. Second, the case enrolled were small because this study was single center study. Multicenter study was needed to increase the cases. Third, the liver etiology such as HBV, HCC, non-viral were different between cases. Fourth, we did not consider the difference of VTQ value between the left and right lobes although VTQ value was significantly higher in the left lobe than those in the right lobe.

In conclusion, elastography with VTQ for FRL before major hepatectomy is an accurate and useful method as a preoperative evaluation. It is more useful indicator to evaluate their values adjusted with the ELRRs.

\section{Acknowledgments}

The authors would like to thank the staff of the Gastroenterological Surgery I Unit of the Hokkaido University Graduate School of Medicine, and Division of Laboratory and Transfusion Medicine Hokkaido University Hospital for their cooperation.

Funding: None.

\section{Footnote}

Conflicts of Interest: All authors have completed the ICMJE uniform disclosure form (available at http://dx.doi. org/10.21037/qims-20-1073). The authors have no conflicts of interest to declare.

Ethical Statement: This study was approved by our institutional review board (approval 17-0335). Informed consent was obtained in the opt-out form on the website of Hokkaido University Hospital.

Open Access Statement: This is an Open Access article distributed in accordance with the Creative Commons Attribution-NonCommercial-NoDerivs 4.0 International License (CC BY-NC-ND 4.0), which permits the noncommercial replication and distribution of the article with the strict proviso that no changes or edits are made and the original work is properly cited (including links to both the formal publication through the relevant DOI and the license). See: https://creativecommons.org/licenses/by-nc-nd/4.0/.

\section{References}

1. Spolverato G, Vitale A, Cucchetti A, Popescu I, Marques HP, Aldrighetti L, Gamblin TC, Maithel SK, Sandroussi
C, Bauer TW, Shen F, Poultsides GA, Marsh JW, TM.

Can hepatic resection provide a long-term cure for patients with intrahepatic cholangiocarcinoma? Cancer 2015;121:3998-4006.

2. Shindoh J, Makuuchi M, Matsuyama Y, Mise Y, Arita J, Sakamoto Y, Hasegawa K, Kokudo N. Complete removal of the tumor-bearing portal territory decreases local tumor recurrence and improves disease-specific survival of patients with hepatocellular carcinoma. J Hepatol 2016;64:594-600.

3. Yokoo H, Miyata H, Konno H, Taketomi A, Kakisaka T, Hirahara N, Wakabayashi G, Gotoh M, Mori M. Models predicting the risks of six life-threatening morbidities and bile leakage in 14,970 hepatectomy patients registered in the National Clinical Database of Japan. Medicine (Baltimore) 2016;95:e5466.

4. Skrzypczyk C, Truant S, Duhamel A, Langlois C, Boleslawski E, Koriche D, Hebbar M, Fourrier F, Mathurin P, Pruvot FR. Relevance of the ISGLS definition of posthepatectomy liver failure in early prediction of poor outcome after liver resection: study on 680 hepatectomies. Ann Surg 2014;260:865-70.

5. Iguchi K, Hatano E, Yamanaka K, Tanaka S, Taura K, Uemoto S. Validation of the conventional resection criteria in patients with hepatocellular carcinoma in terms of the incidence of posthepatectomy liver failure and long-term prognosis. Dig Surg 2015;32:344-51.

6. Jin YJ, Lee SH, Cho SG, Kim JH, Lee JW, Lee KY, Shin WY. Prediction of postoperative liver failure using gadoxetic acid-enhanced magnetic resonance imaging in patients with hepatocellular carcinoma. J Gastroenterol Hepatol 2016;31:1349-56.

7. Cescon M, Colecchia A, Cucchetti A, Peri E, Montrone L, Ercolani G, Festi D, Pinna AD. Value of transient elastography measured with FibroScan in predicting the outcome of hepatic resection for hepatocellular carcinoma. Ann Surg 2012;256:706-12.

8. Chong CC, Wong GL, Chan AW, Wong VW, Fong AK, Cheung YS, Wong J, Lee KF, Chan SL, Lai PBS, Chan HLY. Liver stiffness measurement predicts high-grade post-hepatectomy liver failure: A prospective cohort study. J Gastroenterol Hepatol 2017;32:506-14.

9. Castéra L, Vergniol J, Foucher J, Le Bail B, Chanteloup E, Haaser M, Darriet M, Couzigou P, Lédinghen VD. Prospective comparison of transient elastography, Fibrotest, APRI, and liver biopsy for the assessment of fibrosis in chronic hepatitis C. Gastroenterology 2005;128:343-50. 
10. Nightingale K, McAleavey S, Trahey G. Shear-wave generation using acoustic radiation force: in vivo and ex vivo results. Ultrasound Med Biol 2003;29:1715-23.

11. Nishio T, Taura K, Koyama Y, Tanabe K, Yamamoto G, Okuda Y, Ikeno Y, Seo S, Yasuchika K, Hatano E, Okajima H, Kaido T, Tanaka S, Uemoto S. Prediction of posthepatectomy liver failure based on liver stiffness measurement in patients with hepatocellular carcinoma. Surgery 2016;159:399-408.

12. Nanashima A, Sakamoto A, Sakamoto I, Hayashi H, Abo T, Wakata K, Murakami G, Arai J, Wada H, Takagi K, Takeshita H, Hidaka S, To K, Nagayasu T. Usefulness of evaluating hepatic elasticity using artificial acoustic radiation force ultrasonography before hepatectomy. Hepatol Res 2014;44:1308-19.

13. Pissaia A Jr, Borderie D, Bernard D, Scatton O, Calmus Y, Conti F. APRI and FIB-4 scores are useful after liver transplantation independently of etiology. Transplant Proc 2009;41:679-81.

14. Ferraioli G, Wong VWS, Castera L, Berzigotti A, Sporea I, Dietrich CF, Choi BI, Wilson SR, Kudo M, Barr RG. Liver ultrasound elastography: an update to the World Federation for Ultrasound in Medicine and Biology guidelines and recommendations. Ultrasound Med Biol 2018;44:2419-40.

15. Kubo K, Kawakami H, Kuwatani M, Nishida M, Kawakubo K, Kawahata S, Taya Y, Kubota Y, Amano T, Shirato H, Sakamoto N. Liver elasticity measurement before and after biliary drainage in patients with obstructive jaundice: a prospective cohort study. BMC Gastroenterol 2016;16:65.

16. Yoshitani T, Asakawa N, Sakakibara M, Noguchi K, Tokuda Y, Kamiya K, Iwano H, Yamada S, Kudou Y, Nishida M, Shimizu C, Amano T, Tsutsui H. Value of virtual touch quantification elastography for assessing liver congestion in patients with heart failure. Circ J 2016;80:1187-95.

17. Kawagishi N, Suda G, Kimura M, Maehara O, Shimazaki T, Yamada R, Kitagataya T, Shigesawa T, Suzuki K, Nakamura A, Ohara M, Umemura M, Nakai M, Sho T, Natsuizaka M, Morikawa K, Ogawa K, Kudo Y, Nishida M, Sakamoto N. High serum angiopoietin-2 level predicts non-regression of liver stiffness measurement-based liver fibrosis stage after direct-acting antiviral therapy for hepatitis C. Hepatol Res 2020;50:671-81.

18. Kamiyama T, Nakanishi K, Yokoo H, Kamachi H, Tahara M, Yamashita K, Taniguchi M, Shimamura T, Matsushita M, Todo S. Perioperative management of hepatic resection toward zero mortality and morbidity: analysis of 793 consecutive cases in a single institution. J Am Coll Surg 2010;211:443-9.

19. Fukushima K, Fukumoto T, Kuramitsu K, Kido M, Takebe A, Tanaka M, Itoh T, Ku Y. Assessment of ISGLS definition of posthepatectomy liver failure and its effect on outcome in patients with hepatocellular carcinoma. J Gastrointest Surg 2014;18:729-36.

20. Céspedes I, Ophir J, Ponnekanti H, Maklad N. Elastography: elasticity imaging using ultrasound with application to muscle and breast in vivo. Ultrason Imaging 1993;15:73-88.

21. Krouskop TA, Wheeler TM, Kallel F, Garra BS, Hall T. Elastic moduli of breast and prostate tissues under compression. Ultrason Imaging 1998;20:260-74.

22. Sandrin L, Fourquet B, Hasquenoph JM, Yon S, Fournier C, Mal F, Christidis C, Ziol M, Poulet B, Kazemi F, Beaugrand M, Palau R. Transient elastography: a new noninvasive method for assessment of hepatic fibrosis. Ultrasound Med Biol 2003;29:1705-13.

23. Boursier J, Isselin G, Fouchard-Hubert I, Oberti F, Dib N, Lebigot J, Bertrais S, Gallois Y, Calès P, Aubé C. Acoustic radiation force impulse: a new ultrasonographic technology for the widespread noninvasive diagnosis of liver fibrosis. Eur J Gastroenterol Hepatol 2010;22:1074-84.

24. Galati G, De Vincentis A, Gallo P, Guidi A, VespasianiGentilucci U, Picardi A. Diagnostic value of Virtual Touch Quantification (VTQ®) for differentiation of hemangiomas from malignant focal liver lesions. Med Ultrason 2019;21:371-6.

25. Sugiura R, Kuwatani M, Nishida M, Hirata K, Sano I, Kato S, Kawakubo K, Nakai M, Sho T, Suda G, Morikawa K, Ogawa K, Sakamoto N. Correlation between liver elasticity by ultrasound elastography and liver functional reserve. Ultrasound Med Biol 2019;45:2704-12.

26. Hirashita T, Ohta M, Iwashita Y, Iwaki K, Uchida H, Yada K, Matsumoto T, Kitano S. Risk factors of liver failure after right-sided hepatectomy. Am J Surg 2013;206:374-9.

27. Han H, Hu H, Xu YD, Wang WP, Ding H, Lu Q. Liver failure after hepatectomy: A risk assessment using the prehepatectomy shear wave elastography technique. Eur J Radiol 2017;86:234-40.

28. Tsuruga Y, Kamiyama T, Kamachi H, Shimada S, Wakayama K, Orimo T, Kakisaka T, Yokoo H, Taketomi A. Significance of functional hepatic resection rate calculated using 3D CT/(99m)Tc-galactosyl human serum albumin single-photon emission computed tomography fusion 
imaging. World J Gastroenterol 2016;22:4373-9.

29. Ooi GJ, Clouston A, Johari Y, Kemp WW, Roberts SK, Brown WA, Burton PR. Evaluation of the histological variability of core and wedge biopsies in nonalcoholic fatty liver disease in bariatric surgical patients. Surg Endosc 2021;35:1210-8

30. Toshima T, Shirabe K, Takeishi K, Motomura T, Mano Y, Uchiyama H, Yoshizumi T, Soejima Y, Taketomi A, Maehara Y. New method for assessing liver fibrosis based on acoustic radiation force impulse: a special reference to

Cite this article as: Shimada S, Kamiyama T, Kakisaka T, Orimo T, Nagatsu A, Asahi Y, Sakamoto Y, Kamachi H, Kudo Y, Nishida M, Taketomi A. The impact of elastography with virtual touch quantification of future remnant liver before major hepatectomy. Quant Imaging Med Surg 2021;11(6):25722585. doi: 10.21037/qims-20-1073 the difference between right and left liver. J Gastroenterol 2011;46:705-11.

31. Bachellier P, Rosso E, Pessaux P, Oussoultzoglou E, Nobili C, Panaro F, Jaeck D. Risk factors for liver failure and mortality after hepatectomy associated with portal vein resection. Ann Surg 2011;253:173-9.

32. Zhang LX, Lv Y, Xu AM, Wang HZ. The prognostic significance of serum gamma-glutamyltransferase levels and AST/ALT in primary hepatic carcinoma. BMC Cancer 2019;19:841. 